
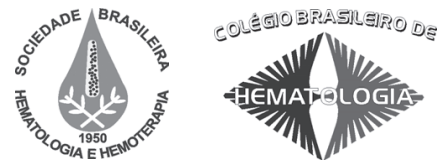

Relato de Caso / Case Report

\title{
Apresentação incomum de sarcoma granulocítico mamário
}

\section{Unusual presentation of granulocytic sarcoma in the breasts}

Francisco D. Rocha Filho ${ }^{1}$

Francisco V. A. Ferreira ${ }^{2}$

Gabrielle G. Lima ${ }^{3}$

Michelle G. Lima ${ }^{3}$

Antônio A. F. Figueiredo ${ }^{3}$

\begin{abstract}
O termo sarcoma granulocítico (SG) designa um raro tumor sólido composto de agregados de precursores granulocíticos imaturos em sítios extramedulares. A lesão geralmente ocorre durante o curso natural da leucemia mieloide aguda (LMA) ou após sua remissão. O SG primário manifesta-se mais comumente na pele e linfonodos, portanto, quando se apresenta na mama, o erro diagnóstico de linfoma não Hodgkin, carcinoma lobular, sarcoma e melanoma maligno é um problema comum. A mama tem sido relatada como um local incomum de SG. Relata-se um caso raro de SG bilateral em mamas concomitante com LMA numa mulher de 47 anos. A paciente foi admitida em nosso hospital devido a manifestações neurológicas e descobrimos, durante a investigação, tumorações nas mamas. A histopatologia das lesões sugeriu linfoma não Hodgkin, sendo iniciada quimioterapia esquema CHOP. No entanto, o mielograma mostrou hiperplasia das séries granulocíticas, e a imuno-histoquímica revelou mieloperoxidase e CD68 positivos, confirmando o diagnóstico de $S G$ primário em mamas. A citogenética não detectou anomalias. A revisão da microscopia e a análise do líquor confirmaram a presença de infiltração no parênquima mamário e no sistema nervoso central por leucemia monoblástica aguda (LMA-M5a). O protocolo de indução da remissão foi iniciado com daunorrubicina, arabinosídeo-C e quimioterapia intratecal com metotrexate, arabinosídeo-Ce dexametasona (MADIT). Um mês depois, a paciente recusou a continuação do tratamento, depois de ter feito pedido de alta. Rev. Bras. Hematol. Hemoter. 2009;31(4):295-298.
\end{abstract}

Palavras-chave: Sarcoma granulocítico; mama; leucemia mieloide aguda.

\section{Introdução}

Sarcoma granulocítico (SG) é raro tumor sólido extramedular composto por células imaturas derivadas de séries hematopoéticas mieloides. ${ }^{1}$ É geralmente associado com leucemia ou outras desordens mieloproliferativas, mas também pode ocorrer sem envolvimento de doenças hematológicas. ${ }^{2}$ SG ocorre em 3\% a 7\% dos casos de leucemia mieloide aguda (LMA). ${ }^{1}$

O SG primário manifesta-se mais comumente na pele e linfonodos, portanto, quando se apresenta na mama, o erro diagnóstico é um problema comum. ${ }^{3}$ A mama é relatada como um local raro de $\mathrm{SG}^{4}{ }^{4}$ Wong et al. mostraram que quase metade dos pacientes com SG primário teve um diagnóstico inicial errado $^{3}$ de linfoma ou sarcoma. ${ }^{5}$ Vários estudos têm abordado as características clínico-patológicas e imuno-histoquímica destas neoplasias. ${ }^{6}$ A maioria dos casos relatados de SG com LMA tem cromossomo translocação $t(8 ; 21){ }^{7}$

$\mathrm{O}$ artigo relata a história de uma paciente que foi admitida em nosso hospital para investigação de um quadro neurológico, sendo descobertas massas bilaterais em mamas. Trata-se de um caso de sarcoma granulocítico primário conco-

\footnotetext{
${ }^{l}$ Médico Patologista - Instituto do Câncer do Ceará - Fortaleza-CE.

${ }^{2}$ Médico Patologista.

Correspondência: Gabrielle Gurgel Lima

Universidade Federal do Ceará

Rua Monsenhor Furtado 1438/ 101-103 - Rodolfo Teófilo

60430350 - Fortaleza-CE - Brasil

Fax: (55 85) 242-3241

E-mail:gabi_gurgel@yahoo.com.br

Doi: $10.1590 / S 1516-84842009005000063$
}

${ }^{3}$ Aluna do Curso de Medicina da Universidade Federal do Ceará - Fortaleza-CE.

Instituto do Câncer do Ceará - Universidade Federal do Ceará - Fortaleza-CE. 

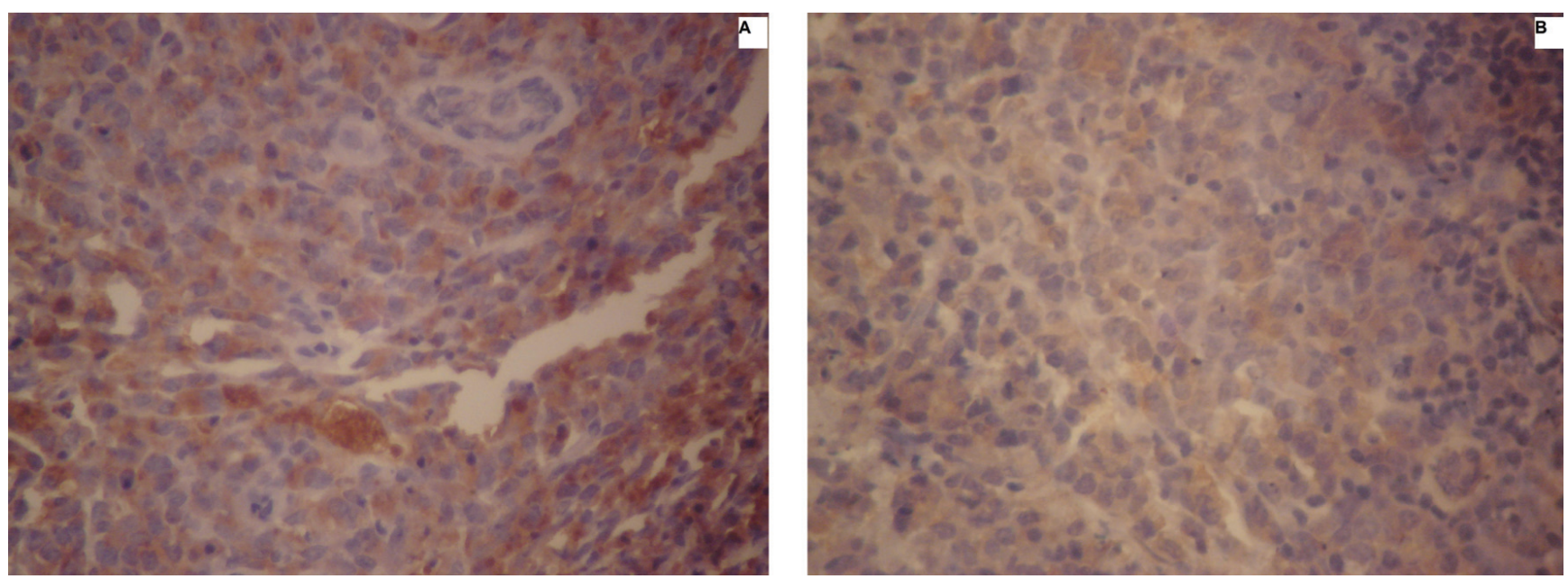

Figura 1. Imuno-histoquímica: A -CD68 positivo; B - mieloperoxidase positivo (400x)

mitante com leucemia mieloide aguda, que foi inicialmente diagnosticado e tratado como linfoma não Hodgkin. Este caso de tumor raro sugere que este diagnóstico diferencial deve ser considerado quando linfoma maligno de mama é detectado.

\section{Relato do Caso}

Paciente do sexo feminino, 47 anos, relatou que em julho de 2005 iniciou quadro de lombalgia contínua, de leve intensidade, com irradiação para membros inferiores, evoluindo em 15 dias para paraplegia. Ao exame físico, apresentava quadro neurológico compatível com neuropatia periférica padrão em bota, arreflexia, paraparesia distal e atrofia de membros inferiores, não deambulando nem se mantendo em posição ortostática. Foi realizada ressonância nuclear magnética (RNM) da coluna lombar, que revelou protusão discal medial ao nível L5-S1, associada a massa sólida preenchendo o saco dural ao nível L4-L5 e lesões focais hipodensas nos corpos vertebrais de L1 e L5, sugestivas de implantes secundários. $\mathrm{O}$ tratamento cirúrgico não pode ser realizado, devido à evidência de massas bilaterais em mamas. De acordo com a análise clínica e a RNM, o quadro neurológico não sugeriu compressão medular. O estudo histopatológico das tumorações das mamas mostrou infiltração linfoide atípica no parênquima mamário sugestiva de linfoma não Hodgkin. Iniciaram-se dois ciclos quimioterápicos com ciclofosfamida, doxorrubicina, vincristina e prednisona (CHOP).

O mielograma realizado um mês depois indicou hiperplasia das linhagens granulocíticas e megacariocíticas e plaquetopoese levemente aumentada. A biópsia da medula óssea evidenciou hipercelularidade às custas de todas as séries, sobretudo a granulocítica. Notaram-se micromegacariócitos com a trama de reticulina aumentada, levando a uma interpretação diagnóstica consistente com infiltração medular por doença mieloproliferativa. A imuno-histoquímica

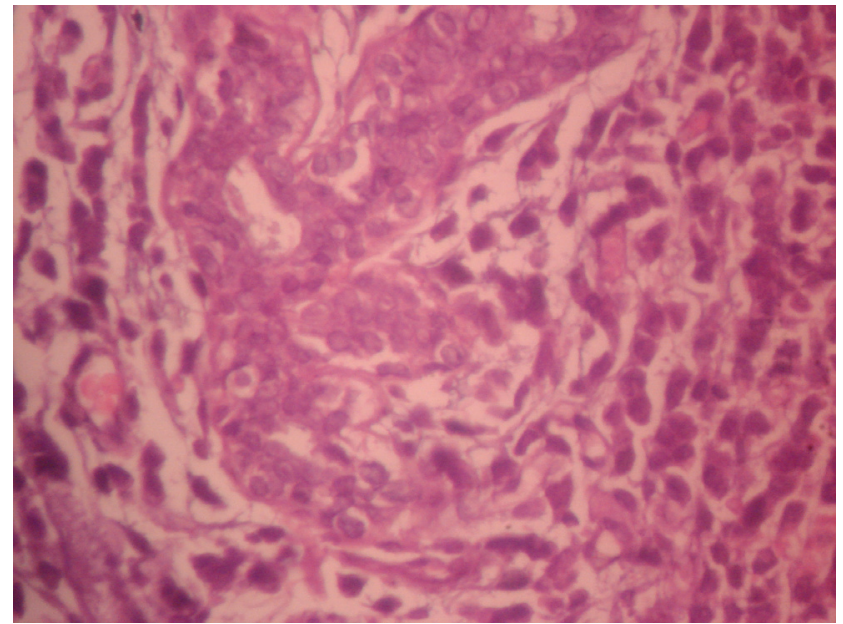

Figura 2. Sarcoma granulocítico\&nbsp; envolvendo ducto mamário\&nbsp; (400x)

das lesões mamárias revelou mieloperoxidase e CD68 positivos (Figura 1), confirmando o diagnóstico de sarcoma granulocítico primário concomitante com leucemia mieloide aguda (LMA). Foram realizadas revisão de lâmina com estudo morfológico e imuno-histoquímico e análise do líquor, que confirmaram a presença de infiltração em mamas e sistema nervoso central por leucemia monoblástica (LMA-M5a). Como foram realizados dois ciclos quimioterápicos, houve remissão da doença no mielograma, porém não no sistema nervoso central. Deu-se início então ao protocolo de indução de remissão da LMA-M5a com daunorrubicina, arabinosídeo$\mathrm{C}$ e dois ciclos de quimioterapia intratecal com metotrexate, arabinosídeo-C e dexametasona (MADIT). A citologia do líquor evidenciou células de médio porte com cromatina frouxa, sobra de nucléolos, exibindo projeções citoplasmáticas.

A citogenética do aspirado de medula óssea não detectou anomalias (cariótipo 46,XX[20]). Um mês depois, a 
paciente, ainda sem deambular, recusou continuação do tratamento, tendo feito pedido de alta.

\section{Conclusão}

O SG que se apresenta como uma massa na mama é raro, com apenas 61 casos relatados na literatura. ${ }^{8}$ Estes tumores representam, na maioria das vezes, recaída ou apresentação inicial da LMA. ${ }^{8}$ Esta lesão primária extramedular pode representar um dilema diagnóstico e terapêutico, tanto para hemopatologistas quanto para oncologistas., 10 Ele pode vir após, concomitante ou previamente à LMA. ${ }^{9}$ No nosso caso, SG das mamas bilateral foi concomitante com LMA.

O SG é relatado em todas as faixas etárias, mas ocorre mais comumente em adultos jovens, com idade média de 37,3 anos (variação de 12-79 anos). A idade de apresentação na mama varia entre os 31-73 anos, com uma média de 52 anos. ${ }^{11}$ Nossa paciente tinha 47 anos de idade. Clinicamente, o SG envolvendo a mama pode se apresentar como uma lesão envolvendo uma ou ambas as mamas. ${ }^{11}$ Nesse caso, os tumores foram bilaterais. Os pacientes são geralmente mulheres, como foi aqui relatado, embora tenha sido encontrado também em indivíduos do sexo masculino, que se apresentavam com ginecomastia. $^{12}$

O SG também é relativamente comum em certos tipos de LMA com anomalias citogenéticas, incluindo com maturação e t $(8 ; 21)(q 22 ;$ q22), leucemia aguda mielomonocítica com eosinófilos com inv ${ }^{16}(\mathrm{p} 13 \mathrm{q} 22)$ ou t(16;16)(p13; q22), e LMA associado com anormalidades $11 \mathrm{q} 23 .{ }^{13}$ Neste caso, a citogenética não detectou anomalias.

Estudos mamográficos têm demonstrado que as lesões podem ter tanto bordas regulares quanto irregulares, com aumento da densidade estromal e não associadas a microcalcificações. Estudos ultrassonográficos de sarcoma mieloide mamário mostraram áreas homogeneamente hipoecoicas com margens bem ou mal definidas. Estudos de imagens de tomografia computadorizada e RNM mostraramm sinais de densidades heterogêneas, mas estes são sinais geralmente hiperdensos quando comparados com os tecidos moles circundantes. Essa aparência variável da imagem torna difícil distinguir SG do carcinoma mamário ou tumores metas-

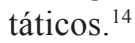

O diagnóstico diferencial deve incluir todos os tumores com proliferação difusa de pequenas células tumorais, como, por exemplo, linfoma não Hodgkin (LNH), carcinomas e melanomas. ${ }^{11}$ Entre os carcinomas, o lobular e o indiferenciado entraram no diagnóstico diferencial. ${ }^{4}$ Condições não neoplásicas, como inflamação e hemopoese extramedular, também devem ser consideradas. ${ }^{4}$ No nosso caso, também o diagnóstico inicial foi de LNH. Somente após a imuno-histoquímica, com positividade para mieloperoxidase e CD68, o diagnóstico de SG foi feito, sendo a paciente tratada com quimioterapia para LMA.
De particular importância na mama, os casos de sarcoma mieloide também podem ser confundidos com neoplasias primárias da mama, em especial o carcinoma lobular invasivo. ${ }^{11}$ Os principais sítios de ocorrência são normalmente linfonodos, pele, intestino delgado e sistema nervoso central, mas a mama é um local incomum de apresentação. ${ }^{5,10}$

O correto diagnóstico histológico é baseado na identificação das características das células granulocíticas no sarcoma. Apesar da característica aparência histológica, há espaço para o erro diagnóstico, e o SG mamário pode ser mais comum do que os poucos relatos de casos que a literatura pode sugerir. ${ }^{15}$ Somente depois pode ser possível estabelecer a verdadeira incidência desta inusitada causa de massa mamária.

Estudos imuno-histoquímicos são extremamente úteis para reconhecer sarcoma mieloide. ${ }^{5}$ Métodos citoquímicos, tais como a coloração mieloperoxidase, podem fornecer provas úteis da evidência da origem mielogênica das células. ${ }^{15}$ Mieloperoxidase é um dos mais específicos marcadores de diferenciação mieloide. CD68 também é positivo em cerca de dois terços dos casos de sarcoma mieloide e é particularmente útil na avaliação de neoplasias monocíticas. ${ }^{6}$ No presente estudo, ambos os marcadores foram positivos.

\begin{abstract}
Granulocytic sarcoma (GS) is an uncommon solid tumor composed of aggregates of immature granulocytic precursors in extramedullary sites. The lesion generally occurs during the natural course of acute myelogenous leukemia or after remission has been achieved. Primary GS manifests most commonly in skin and lymph nodes, therefore when it presents in the breast, misdiagnosis of non-Hodgkin's lymphoma, lobular carcinoma, sarcoma and malignant melanoma is a common problem. The breast has been reported to be an uncommon site for GS. We report on a rare case of granulocytic sarcoma presenting as bilateral breast masses concomitant with acute myeloid leukemia in a 47-year-old woman. The patient was admitted to our hospital due to neurological manifestations, at which time we discovered lesions in the breasts. The histopathology suggested non-Hodgkin lymphoma, and chemotherapy using the CHOP regimen was performed. However, a myelogram showed hyperplasia of the granulocyte cells and immunohistochemistry tests were positive for myeloperoxidase and CD68, confirming the diagnosis of primary granulocytic sarcoma of the breasts. Cytogenetic examinations did not detect anomalies. The review of microscopy and the analysis of cerebrospinal fluid confirmed the presence of infiltration in the breast and in the central nervous system by acute monoblastic leukemia (AML M5a). AML M5 a protocols had been started with daunorubicin, arabinoside$C$ and intrathecal chemotherapy using methotrexate, arabinoside$C$ and dexamethasone (MADIT). One month later, the patient refused further treatment. Rev. Bras. Hematol. Hemoter. 2009; 31(4):295-298.
\end{abstract}

Key words: Granulocytic sarcoma; breast; acute myeloid leukemia. 


\section{Referências Bibliográficas}

1. Khan MY, Hussein KK, Walter MG, Hasan MK, Kern W, KharfanDabaja MA. Granulocytic sarcoma presenting with malignant anasarca in a patient with secondary acute myeloid leukemia. Int J Hematol. 2004;79(3):250-2.

2. Ramasamy K, Lim Z, Pagliuca A, Devereux S, Ho AY, Mufti GJ. Acute myeloid leukaemia presenting with mediastinal myeloid sarcoma: report of three cases and review of literature. Leuk Lymphoma. 2007;48(2):290-4.

3. Wong WS, Loong F, Ooi GC, Tse TC, Chim CS. Primary granulocytic sarcoma of the mediastinum. Leuk Lymphoma. 2004;45(9): 1931-3.

4. Ngu IW, Sinclair EC, Greenaway S, Greenberg ML. Unusual presentation of granulocytic sarcoma in the breast: a case report and review of the literature. Diagn Cytopathol. 2001;24(1):53-7.

5. Shea B, Reddy V, Abbitt P, Benda R, Douglas V, Wingard J. Granulocytic sarcoma (chloroma) of the breast: a diagnostic dilemma and review of the literature. Breast J. 2004;10(1):48-53.

6. Chen J, Yanuck RR 3rd, Abbondanzo SL, Chu WS, Aguilera NS. c-Kit (CD117) reactivity in extramedullary myeloid tumor/granulocytic sarcoma. Arch Pathol Lab Med. 2001;125(11):1448-52.

7. Colovic N, Colovic M, Cemerikic V, Terzic T, Ivanovic S, Skender $\mathrm{M}$, et al. Granulocytic sarcoma of the brain in a patient with acute myeloid leukemia. Acta Chir Iugosl. 2004;51(3):129-31.

8. D'Costa GF, Hastak MS, Patil YV. Granulocytic sarcoma of breast: an aleukemic presentation. Indian J Med Sci. 2007;61(3):152-5.

9. Breccia M, Petti MC, Fraternali-Orcioni G, Monarca B, Latagliata R, D'Elia GM, et al. Granulocytic sarcoma with breast and skin presentation: a report of a case successfully treated by local radiation and systemic chemotherapy. Acta Haematol. 2000; 104(1):34-7.

10. Quintas-Cardama A, Fraga M, Antunez J, Forteza J. Primary extramedullary myeloid tumor of the breast: a case report and review of the literature. Ann Hematol. 2003;82(7):431-4.

11. Valbuena JR, Admirand JH, Gualco G, Medeiros LJ. Myeloid sarcoma involving the breast. Arch Pathol Lab Med. 2005;129(1):32-8.

12. Au WY, Ma SK, Kwong YL, Lie AK, Shek WH, Chow WC, et al. Acute myeloid leukemia relapsing as gynecomastia. Leuk Lymphoma. 1999;36(1-2):191-4.

13. Brunning RD, Matutes E, Flandrin G, Vardiman J. Myeloid sarcoma. In: Jaffe ES, Harris NL, Stein H, Vardiman JW, eds. Pathology and Genetics of Tumours of Haematopoietic and Lymphoid Tissues. Lyon, France: IARC Press; 2001:104-105. World Health Organization Classification of Tumours; vol 3.

14. Barloon TJ, Young DC, Bass SH. Multicentric granulocytic sarcoma (chloroma) of the breast: mammographic findings. AJR Am J Roentgenol. 1993;161(5):963-4.

15. O'Donnell JR, Farrell MA. Acute myelogenous leukaemia with bilateral mammary gland involvement. J Clin Pathol. 1980;33 (6):547-51.

Avaliação: Editor e dois revisores externos

Conflito de interesse: sem conflito de interesse

Recebido: 20/11/2008

Aceito após modificações: 02/03/2009 